\title{
Fragrance and Flavor Analyzer Using Odor Deviation Map
}

\author{
Junichi Kita* and Kiyoshi Toko $^{1}$ \\ Shimadzu Corporation, 1 Nisinokyo Kuwabara-cho, Nakagyo-ku, Kyoto 604-8511, Japan \\ ${ }^{1}$ Graduate School of Information Science and Electrical Engineering, Kyushu University, \\ 744 Motooka, Nishi-ku, Fukuoka-shi, Fukuoka 819-0395, Japan
}

(Received April 19, 2013; accepted July 30, 2013)

Key words: electronic nose, metal-oxide semiconductor, nonlinear response, autoconcentration adjustment, odor deviation map

We developed a fragrance and flavor analyzer system that overcomes the drawback of electronic noses using metal-oxide semiconductors, i.e., the nonlinearity of their outputs with respect to the concentration of samples. This was realized by installing a dilution and mixing apparatus and a concentrating system before fragrance and flavor analyses as pretreatment systems, applying feedback to the sensor outputs for each measurement, and adjusting the lengths of vectors comprising the multiple sensor outputs to a certain value in the range where the sensor outputs are relatively linear. When the lengths of the vectors comprising the multiple sensor outputs are adjusted to a certain value for each measurement, the type of odor can be evaluated using the vector direction. The intensity of the odor is calculated from the dilution or concentration rate required to adjust the vector length to a certain value. By modifying this method, we also developed a technique of odor deviation mapping to quantitatively display changes in the type of odor. In odor deviation mapping, a reference odor and one or two odor deviations, which indicate the deviations from the reference odor, are selected while their vector lengths are being adjusted. Deviation axes are prepared on a map using vectors comprising sensor outputs obtained by adding each odor deviation to the reference odor, and the deviations of odors from the reference odor are quantitatively visualized on the map. As examples, applications of the map to the determination of expiration dates of foods and the identification of substances causing unusual odors are also discussed in this paper.

\section{Introduction}

Fragrance and flavor analyzers, also known as electronic noses, have mainly been studied in Europe since their principle was presented by Persaud and Dodd in 1982.(1) Prototype electronic noses were developed around 1993 and became commercially available in 1995..$^{(2,3)}$ In an international conference on electronic noses held in Tübingen, Germany, in 1999, a total of 28 companies and institutions exhibited electronic nose

*Corresponding author: e-mail: j-kita@shimadzu.co.jp 
systems. ${ }^{(4)}$ However, the number of commercial electronic noses has gradually decreased although new systems have occasionally been developed and reported. Currently, electronic noses are manufactured by only a few companies worldwide.

The reasons for the above decrease are considered to be as follows. Although metal-oxide semiconductor and quartz crystal sensors are relatively stable among the sensors used in conventional electronic noses, they have a slightly lower sensitivity than that required for their practical application. In addition, the outputs of metal-oxide semiconductor sensors, which exhibit the highest sensitivity among the above sensors, are nonlinear with respect to the sample concentration, making the practical application of these sensors difficult. Although the low sensitivity can be improved by applying a sample-concentrating method such as the use of a trap tube, the problem of nonlinearity will be worsened, i.e., the nonlinearity of the sensor outputs with respect to the sample concentration will further increase.

Shurmer et al. also pointed out the nonlinearity of the outputs of metal-oxide semiconductor sensors as a drawback and proposed a method of resolving the nonlinearity by modifying the amplifier circuit at the initial stage of their measurement system. ${ }^{(5)}$ However, this method can only be applied to measurements of relatively high-concentration samples using a single sensor. When using electronic noses, which are equipped with multiple sensors and are also required to measure low-concentration samples, it is difficult to eliminate the nonlinearity of the outputs of all sensors.

In general, multivariate analysis is adopted to analyze signals from electronic noses..$^{(6-12)}$ This analysis can be used if the sensitivity of the sensors to odor substances is equivalent to that of olfactory receptors and the sensor outputs are linear with respect to the sample concentration. For metal-oxide semiconductor electronic noses, however, their sensitivity is different from that of olfactory receptors and their outputs are nonlinear with respect to the sample concentration. Therefore, multivariate analysis can only be applied in special cases, meaning that other analytical methods or measures to overcome the problem of sensor nonlinearity are required.

To solve the above problems, an absolute expression method was developed. ${ }^{(13)}$ By this method, vectors comprising multiple sensor outputs are used for analysis, as shown in Fig. 1. The length of the vector is proportional to the intensity of the odor, and the type of odor is expressed by the direction of the vector. However, it was difficult to calculate the angle between two vectors to compare two corresponding odors because the loci of the vectors were curved owing to the nonlinearity of the outputs of the individual metal-oxide semiconductor sensors with respect to the concentrations of the odor substances.

The purpose of this study is to propose a practical odor sensing system that overcomes the above problems. Specifically, a dilution and mixing apparatus was installed before the sensing stage, which consists of multiple sensors, to dilute highconcentration samples by applying feedback to the sensor outputs. A trap tube was also installed before the sensing stage to concentrate low-concentration samples. Because of this structure, the lengths of vectors representing multiple sensor outputs were adjusted to a certain value during the measurement of the samples. Thus, the problem of the nonlinearity of the sensor outputs with respect to the sample concentration 


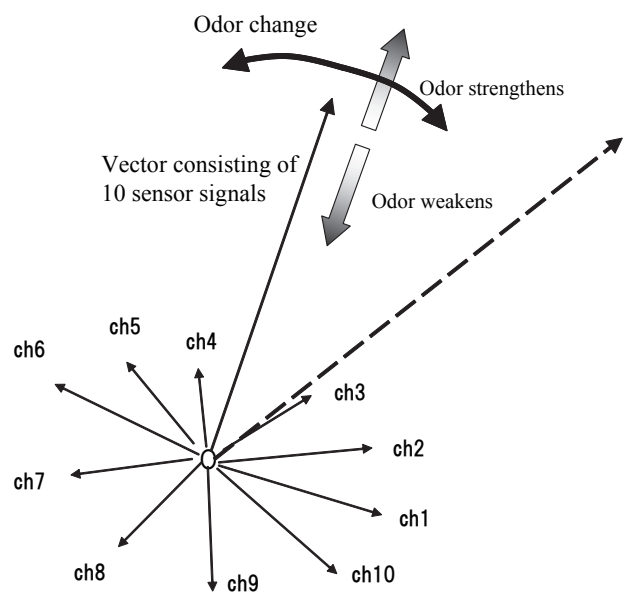

Fig. 1. Principle of the single processing of fragrance and flavor analyzer. "ch" represents the channel of the ten sensors.

was successfully resolved. As a result, the accuracy of odor classification and the repeatability of measurements were improved, enabling the preparation of an odor deviation map with improved accuracy of expressing changes in the type of odor. The odor deviation map can quantitatively visualize changes in the type of odor in terms of multiple components in addition to single components and express a change as that of a multicomponent odor. Therefore, the developed method using odor deviation maps can also be used for odors with a sensory expression, which are generally multicomponent odors, allowing the comparison with sensory tests, enabling the expiration dates of foods to be set, the characterization of food products, and estimation of the causative factors of multicomponent odors.

\section{Materials and Methods}

\subsection{Development of measurement method for adjusting vector length}

An absolute expression method is used to quantify the type and intensity of odors from the sensor outputs for odor samples and a reference gas and enables approximate quantification even when the sensor outputs are nonlinear with respect to the sample concentration. However, the method cannot be used to accurately determine a change in the type of odor in the case of nonlinearity. For example, as shown in Fig. 2, when the concentrations of three samples with slightly different types of odor are gradually changed, each vector corresponding to the sensor outputs forms a curved locus because of the nonlinearity.

As is clear from the figure, the magnitude of the differences among the three odors depends on their concentrations. With decreasing concentration, the differences in the vector angle due to the differences in the type of odor decrease. A similar tendency is 


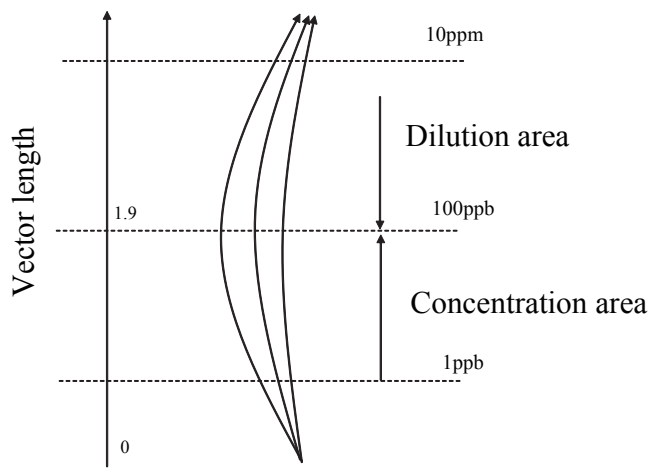

Fig. 2. Adjustment of vector length.

also observed when the concentration becomes excessive. Namely, there is an optimal concentration for classifying different types of odor. Good odor separation ability and good repeatability are realized by unifying the vector length to the suitable length for all measurement samples.

Generally, the shape of the output signals versus concentration is like a sigmoid curve. Before the analysis by using the sensor signals, the normalization of the sensor signals is carried out to average the sensor signals between sensor to sensor. After the normalization, 0.6 is the suitable output level for every 10 sensors. That is the suitable vector length is 1.9 , which is calculated as the square root of the sum of 10 sensor squares.

With this background, we developed a measurement method for adjusting the vector length to resolve the problem of nonlinearity. Specifically, the sensor outputs are monitored and used for feedback to dilute high-concentration samples using a dilution and mixing apparatus (Fig. 3). In the apparatus, a $100 \mathrm{~mL}$ airtight syringe can be slid up and down using a stepping motor, and multiple electromagnetic valves are placed in several channels. The syringe can be heated to $40-90{ }^{\circ} \mathrm{C}$ to prevent the adsorption of gas onto its inner wall.

Low-concentration samples are concentrated using a trap tube containing graphitebased materials (Fig. 4). Odor substances adsorbed on the trap tube at $40{ }^{\circ} \mathrm{C}$ are expelled with the carrier gas generated by heating the trap tube at an arbitrary temperature between 40 and $280{ }^{\circ} \mathrm{C}$. Figure 5 shows the overall configuration of the system. Gaseous odor samples are introduced into sample bags using a sampling pump. Liquid and solid odor samples are placed in sample bags from 0.1 to 10 milliliters and grams, respectively, and left to settle at room temperature for approximately $1 \mathrm{~h}$. Subsequently, only the head space gases are transferred to other bags and used as gaseous odor samples. A certain quantity of each gaseous odor sample collected in the sample bag is introduced into the main syringe and diluted to a predetermined concentration by subsequently introducing nitrogen gas from a syringe containing high-purity nitrogen gas or from a sample bag filled with high-purity nitrogen gas. The sample is diluted by a factor of up to six per dilution to ensure dilution accuracy. The dilution is repeated if the sample needs to be further diluted. 

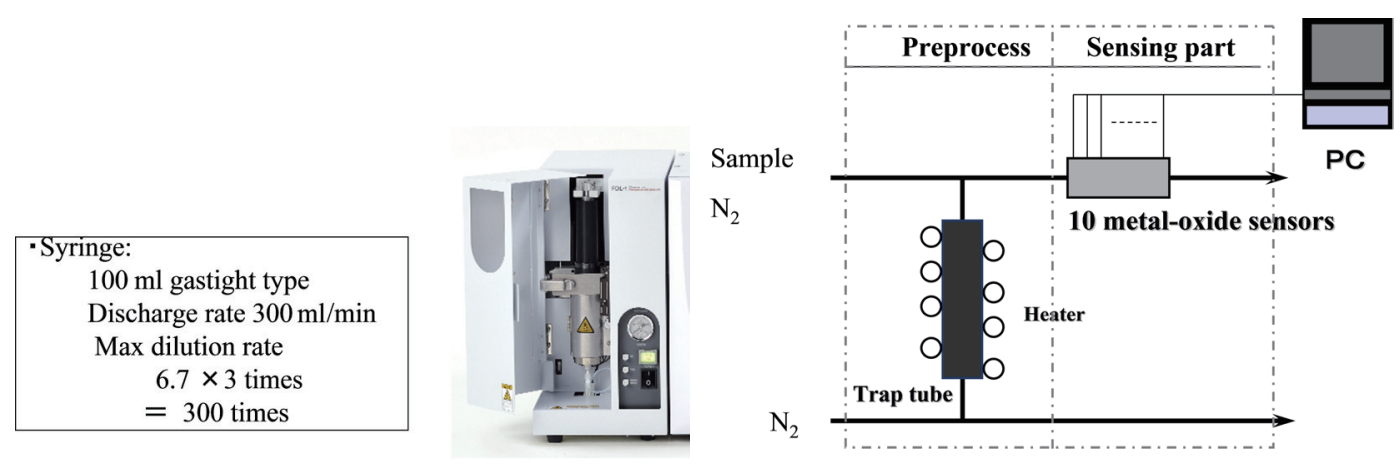

Fig. 3 (left). (Color online) Dilution and mixing apparatus.

Fig. 4 (right). Trap tube used as a preconcentrator.

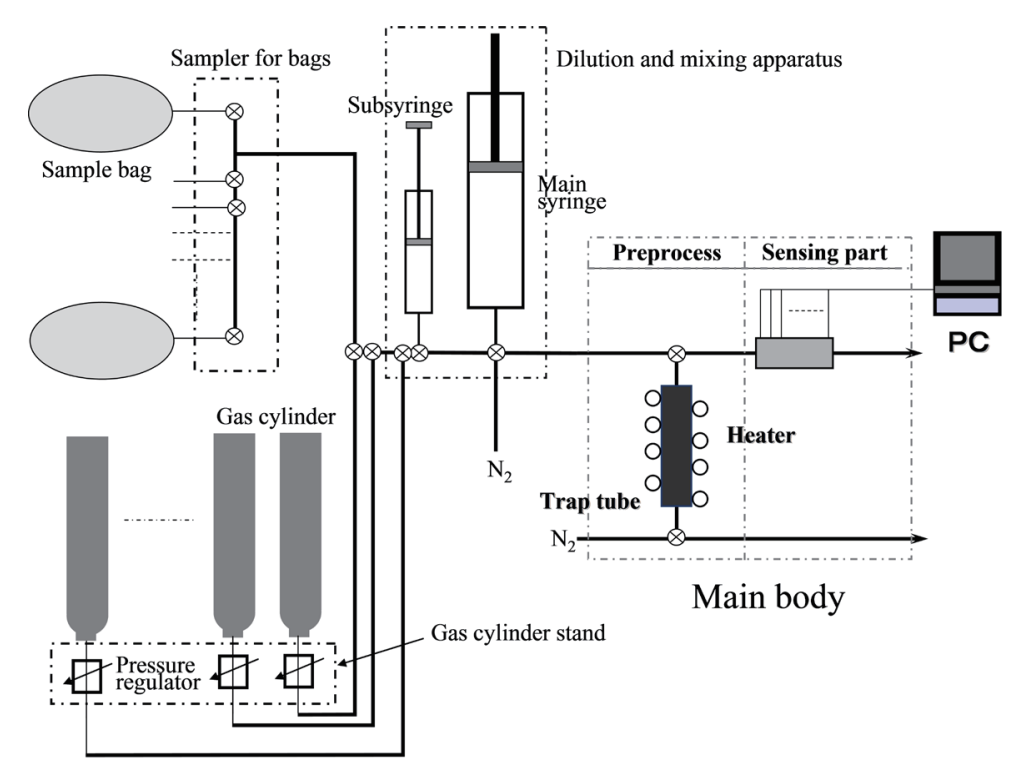

Fig. 5. Device configuration.

Reference gases are introduced using gas cylinders to minimize the effect of the ambient temperature. The inner wall of the aluminum cylinders is treated to prevent the adsorption of gases. The cylinders are filled with a reference gas (in balance with nitrogen) to a maximum pressure of $3 \mathrm{MPa}$. The reference gas is depressurized to 0.1 MPa using a pressure regulator incorporated in a gas cylinder stand and then introduced into the dilution and mixing apparatus. If the gas is introduced into the main syringe in a pressurized state, the concentration of the gas will change by an amount corresponding to the pressure. Therefore, a certain amount of the gas is introduced into a subsyringe to 
restore the ambient pressure and then introduced into the main syringe, which is repeated as required.

Both the odor samples from the sample bags and the reference gases from the gas cylinders are diluted at a predetermined rate for the first measurement to obtain sensor outputs. The measurement is repeated by changing the dilution or concentration rate until the desired sensor output is obtained. The concentration rate is adjusted by changing the amount of gas introduced from the main syringe to the trap tube.

\subsection{Definition of odor deviation}

The types of odor are expressed by two methods: one focuses on the difference in the entire odor such as amine and sulfurous odors, and the other focuses on the difference in a particular odor (for example, the difference in yogurt odor among yogurt samples). The odor analysis of foods frequently adopts the latter method. In this case, for example, a reference yogurt odor is determined and the odor of yogurt samples is expressed in terms of the difference from the reference yogurt odor, which is subjectively carried out by humans. We propose the concept of odor deviation to realize the above-mentioned odor expression method using fragrance and flavor analyzers.

Odor deviation is defined as the deviation of an odor from a reference odor and is equivalent to standard odor expressions corresponding to those used in flavor wheels. ${ }^{(14)}$ The odors of samples may be expressed by adding several odor deviations equivalent to the standard odor to the reference odor, as shown in Fig. 6.

We developed a technique of odor deviation mapping to apply the odor deviations to fragrance and flavor analyzers. In this technique, each of two odor deviations is added to a reference odor positioned at the origin on a map. For fragrance and flavor analyzers, multicomponent odors can be used for the odor deviations, meaning that a map of odors as imagined by humans on a sensory basis can be reproduced using the fragrance and flavor analyzers when odors with a sensory expression used in flavor wheels are used as odor deviations. Moreover, the calibration of odor deviation mapping will become possible by accumulating the results of measurements using common odors for reference

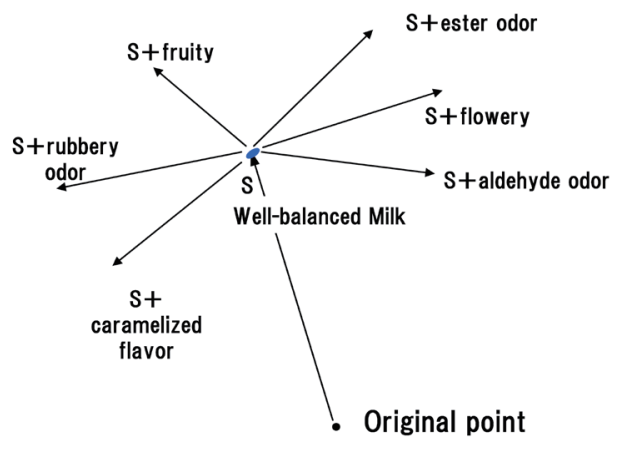

Fig. 6. Principle of odor deviation map. S represents a well-balanced milk sample. 
odors and odor deviations. In addition, some odor deviations (e.g., a charred odor) can be commonly applied to various reference samples, regardless of the type of food material, indicating that the odor deviation mapping has strong potential as a highly versatile method.

\subsection{Technique of odor deviation mapping}

The technique of mapping odor deviations from measured values obtained using a fragrance and flavor analyzer with odor deviations, described in $\S 2.2$, is explained using Fig. 7. First, a reference sample (e.g., normal milk) is selected, and then two odors that may be a cause of the difference from the reference sample (i.e., odor deviations) are selected. In this experiment, foul odor (odor deviation 1) and green odor (odor deviation 2 ) were selected. These odors are generally treated using sample bags but can be treated using gas cylinders as required. Odor deviation 1 is added to the reference sample on a map. This changes the vector direction and concentration of the sample because the reference odor is added with another odor. Although the vector length also changes, it must be adjusted to that of the original reference sample for the reason described in $\S 2.1$, that is to realize good separation ability and good repeatability. Therefore, it is adjusted by diluting the mixed sample using the dilution and mixing apparatus through the feedback of the sensor outputs.

In this experiment, reference and test samples were introduced from sample bags or gas cylinders into the main syringe, and nitrogen gas was introduced from a $20 \mathrm{~L}$ nitrogen gas bag installed at the sampler for the bags into the main syringe to dilute the samples. To add an odor deviation to the reference sample, they were introduced into the dilution and mixing apparatus at a predetermined ratio and diluted by introducing nitrogen gas from the nitrogen gas bag. Odor deviation was also introduced from sample
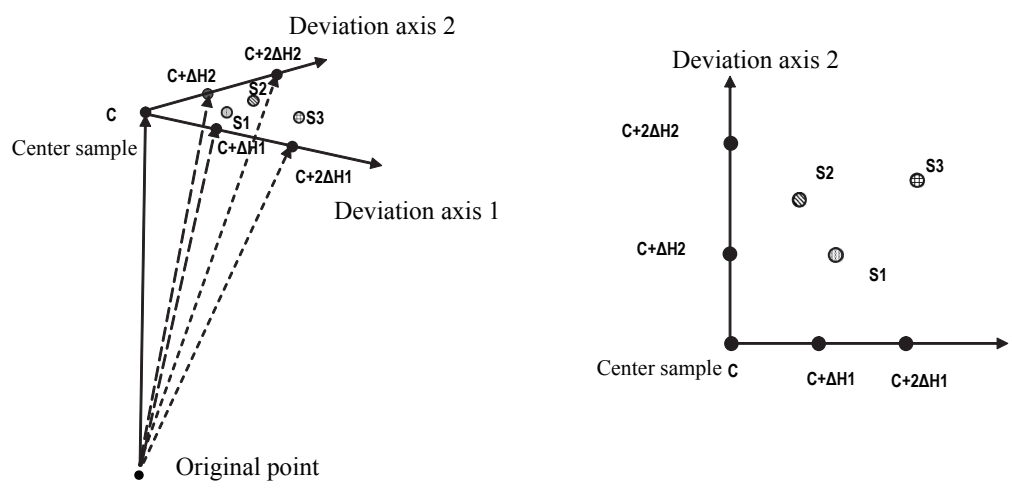

Fig. 7. Construction of odor deviation map. The original point represents the origin of the map. $\mathrm{C}$ represents the reference sample. $\mathrm{C}+\Delta \mathrm{H} 1$ represents the reference sample with a small amount of $\mathrm{H} 1$ added. $\mathrm{C}+2 \Delta \mathrm{H} 1$ represents the reference sample with twice of the amount of $\Delta \mathrm{H} 1$ added. $\Delta \mathrm{H} 2$ has a meaning similar to that $\Delta \mathrm{H} 1$. 
bags or gas cylinders similarly to the reference sample.

The above procedure was performed for both odor deviations 1 and 2 with two different quantities to prepare deviation axes. Subsequently, the reference sample was subjected to treatment to change its odor (for example, heating the reference milk sample to induce its deterioration) and used as a test sample. The obtained data were plotted on the map (S1, S2, and S3 in the figure). Here, deviation axes 1 and 2 were set perpendicular to each other for ease of understanding, although they are generally not perpendicular.

\section{Results and Discussion}

Figure 8 shows an example of automatically adjusting the vector length. In this experiment, the outputs of ten sensors were measured for seven types of gas molecule with different functional groups, and the vector length was adjusted to 1.9, at which all sensors exhibited relatively linear outputs. The table in the figure summarizes the data obtained when the concentration of a sample was adjusted by applying feedback to the measured outputs of the sensors. The dilution rate was gradually decreased because the vector length obtained when the initial dilution rate was set to 20 was much lower than 1.9. The vector length refers to the length of the vector comprising the outputs of the ten sensors. The sensor output is defined by

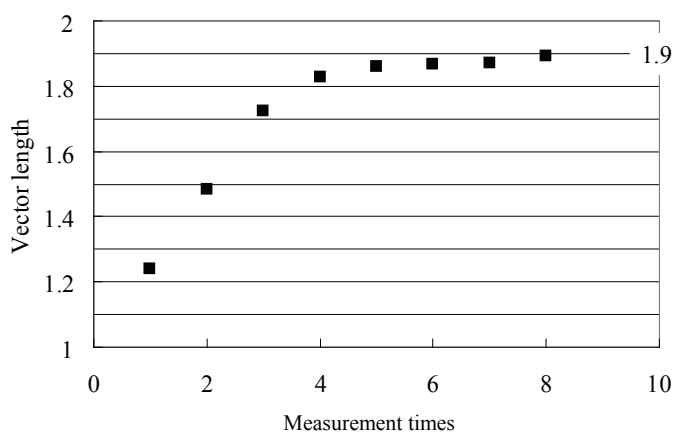

\begin{tabular}{|c|c|c|c|c|c|c|c|c|c|}
\hline & $\begin{array}{l}\text { Target } \\
\text { value }\end{array}$ & 1 & 2 & 3 & 4 & 5 & 6 & 7 & 8 \\
\hline Dilution ratio & & 20 & 5.262 & 2.268 & 1.582 & 1.473 & 1.473 & 1.473 & 1.385 \\
\hline $\begin{array}{l}\text { Total sensor signal } \\
\text { (vector length) }\end{array}$ & & 1.2414 & 1.4849 & 1.7224 & 1.8292 & 1.8602 & 1.867 & 1.8693 & 1.8931 \\
\hline $\begin{array}{l}\text { Vector length deviation } \\
\text { from previous time }\end{array}$ & 0.015 & & & & & & 0.0068 & 0.0023 & \\
\hline $\begin{array}{l}\text { Angle deviation from } \\
\text { previous time }\end{array}$ & 0.25 & & & & & & 0.2876 & 0.1432 & \\
\hline
\end{tabular}

Fig. 8. Adjustment of vector length. 


$$
\text { Sensor output }=-\log \left(R / R_{0}\right) \text {. }
$$

Here, $R_{0}$ and $R$ are the sensor resistances during the measurement of odorless and odor gases, respectively.

It would have taken a very long time to adjust the vector length to exactly 1.9 by applying feedback because the response rates of the sensors are not so high. Therefore, the dilution rate was fixed when a vector length close to 1.9 (within \pm 0.05 ) was obtained, which was achieved at the fifth measurement for the example shown in Fig. 8. The sensor output was judged to have stabilized when the differences in the vector length and angle from those of the previous measurement fell below certain values, as shown in the table. Here, the sensor output was considered to have stabilized when the difference in vector length was 0.015 or smaller (at the sixth measurement) and the difference in vector angle was $0.25^{\circ}$ or smaller (at the seventh measurement). Once the sensor output had stabilized, one more measurement was carried out after adjusting the dilution rate to make the vector length closer to 1.9. The vector length was determined by interpolating the first and second stabilization points.

Figure 9 shows an example of an odor deviation map for milk, which can be used to set its expiration date. Thermally deteriorated milk samples were prepared by heating milk at $40{ }^{\circ} \mathrm{C}$ for different durations. Data obtained from the thermally deteriorated milk samples were plotted on the map, in which normal milk was positioned on the origin, and aldehyde and green odors were used for the deviation axes. These odors were selected from among the six odor deviations developed in this study. As a result, the data obtained from the thermally deteriorated milk samples were distributed on the side of the deviation axis corresponding to the aldehyde odor. Therefore, the odor deviation

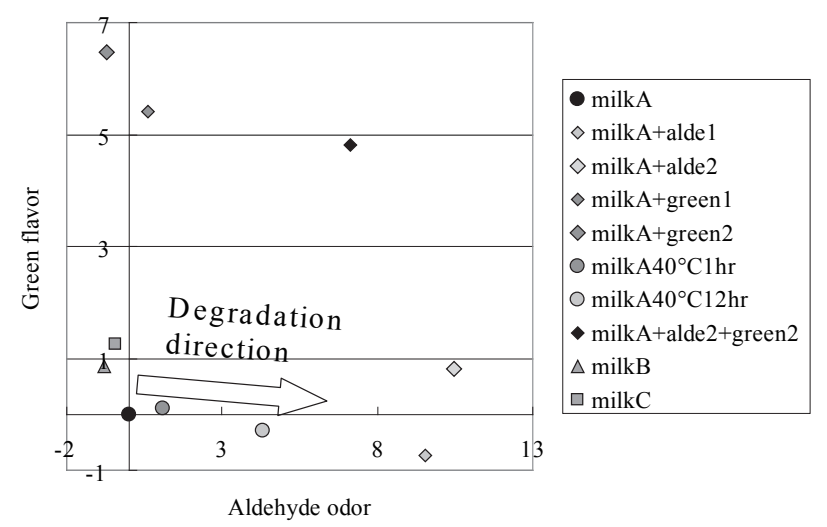

Fig. 9. Odor deviation map of milk. MilkA+1/10alde represents Milk A with aldehyde odor of one-tenth that of Milk A added. MilkA+1/3alde represents Milk A with aldehyde odor of one-third that of Milk A added. For green odors, similar expressions are used. MilkA $\left(40{ }^{\circ} \mathrm{C}, 1 \mathrm{~h}\right)$ represents Milk A heated at $40{ }^{\circ} \mathrm{C}$ for $1 \mathrm{~h}$ and MilkA $\left(40{ }^{\circ} \mathrm{C}, 12 \mathrm{~h}\right)$ represents Milk A heated at $40{ }^{\circ} \mathrm{C}$ for 12 h. MilkA+1/3alde+1/3green represents Milk A with aldehyde and green odors both with one-third that of Milk A added. Milks B and C are test samples. 
map can be used to visualize and calibrate changes in the odor of milk on the basis of the amount of added aldehyde odor, in contrast to the absolute expression method, which cannot cancel out the change in sensor output with time. ${ }^{(13)}$

In odor deviation mapping, measurements are carried out while adjusting the vector length for all measurement data, and the sensor outputs for both the reference odor and odor deviations are measured for each measurement. This enables the cancellation of any change in sensor output with time, enabling us to detect slight changes in the type of odor, which may be hidden by the drift of sensor output in conventional methods, as shown in Fig. 10. Here, among the odor deviations developed in this study, a fruity odor was used as the reference odor, and green and aldehyde odors were used for deviation axes 1 and 2, respectively. In addition, samples obtained by adding green and aldehyde odors with ratios of 3:7 and 7:3 were used as test samples. The sensor outputs were measured for the reference odor, odor deviations, and test samples in this order, which was performed twice. The results of the two measurements were plotted on a map. In Fig. 10, capital and lower-case letters represent the results of the first and second measurements, and F, G, and A represent fruity, green, and aldehyde odors, respectively. $F+G 1$ and $F+G 2$ represent $F$ with amounts of $G$ equal to one-tenth and one-third that of F, respectively. Similarly, F+A1 and F+A2 represent F with amounts of A equal to one-tenth and one-third that of F, respectively. Figure 10(a) shows the measurement results obtained by automatically adjusting the vector length using the dilution and mixing apparatus shown in Fig. 3, revealing good agreement between the

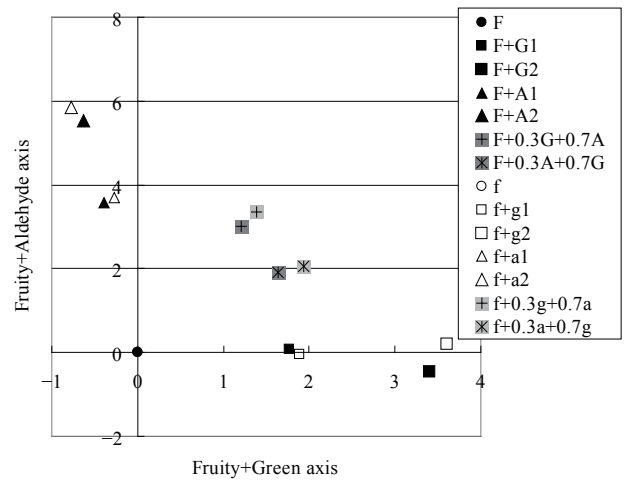

(a)

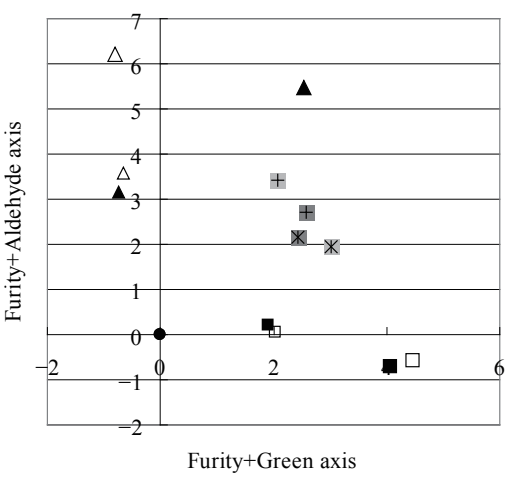

(b)

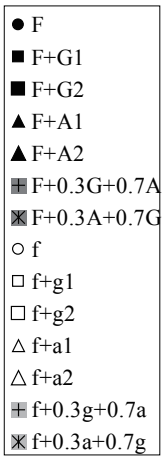

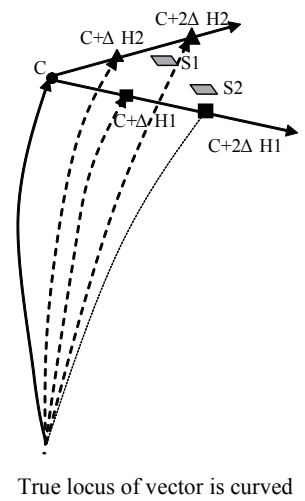

(c)

Fig. 10. Repeatability of odor deviation map. (a) Diluted by apparatus and (b) diluted by hand. $\mathrm{F}, \mathrm{G}$, and A represent fruity, green, and aldehyde odors, respectively. $\mathrm{F}+\mathrm{G} 1$ and $\mathrm{F}+\mathrm{G} 2$ represent $\mathrm{F}$ with amounts of $\mathrm{G}$ equal to one-tenth and one-third that of $\mathrm{F}$, respectively. Similarly, $\mathrm{F}+\mathrm{A} 1$ and $\mathrm{F}+\mathrm{A} 2$ represent $\mathrm{F}$ with amounts of $\mathrm{A}$ equal to one-tenth and one-third that of $\mathrm{F}$, respectively. $\mathrm{F}+0.3 \mathrm{G}+0.7 \mathrm{~A}$ represents $\mathrm{F}$ with $30 \% \mathrm{G}$ and $70 \% \mathrm{~A}$ added. Capital and lower-case letters represent the results of the first and second measurements, respectively. S1 and S2 in (c) represent test samples (central, fruity flavor; deviation 1, green odor; deviation 2, aldehyde odor). 
two measurements. All of the deviations from the 1 st time to the 2 nd time are less than $0.3^{\circ}$. For comparison, Fig. 10(b) shows the measurement results obtained by manually adjusting the concentrations of samples using syringes and sample bags so that as similar sensor outputs as possible are obtained among the samples. The differences between the two measurements, approximately $0.5^{\circ}$ beside the bad case of $3^{\circ}$, were greater than those in the case of automatic adjustment using the dilution and mixing apparatus.

This indicates that, unless the vector length is accurately adjusted, the directions of vectors will deviate from that of the reference vector because of the curved vector loci resulting from the nonlinearity of sensor outputs.

Figure 11 shows an odor deviation map of commercially available vegetable juices and the result of estimating an unusual odor in a multicomponent odor as an application of odor deviation mapping. Among the commercially available vegetable juices, the one with the most typical odor was selected as the reference sample ( $\mathrm{T}$ in the figure), and the juices with the strongest vegetable and fruity odors were selected as samples used for deviation axes 1 and 2, respectively. In the figure, $\mathrm{VC}$ represents samples with a strong vegetable odor (i.e., "vegetable group") and the number after VC (1, 2, and 3) represents the sample number in the group. Similarly, FC represents samples with a strong fruity odor (i.e., "fruity group"), MC represents samples with a well-balanced odor of vegetables and fruit (i.e., "mixed-odor group"), and $\mathrm{ViC}$ represents a sample in which vitamin juice is mixed with vegetable juice. The figure provides an easy-to-understand classification of the samples, indicating, for example, samples with a more vegetable or a more fruity odor. Samples with an odor other than those used for the deviation axes, such as $\mathrm{ViC}$, are plotted far from the axes. These results indicate that the deviation axes
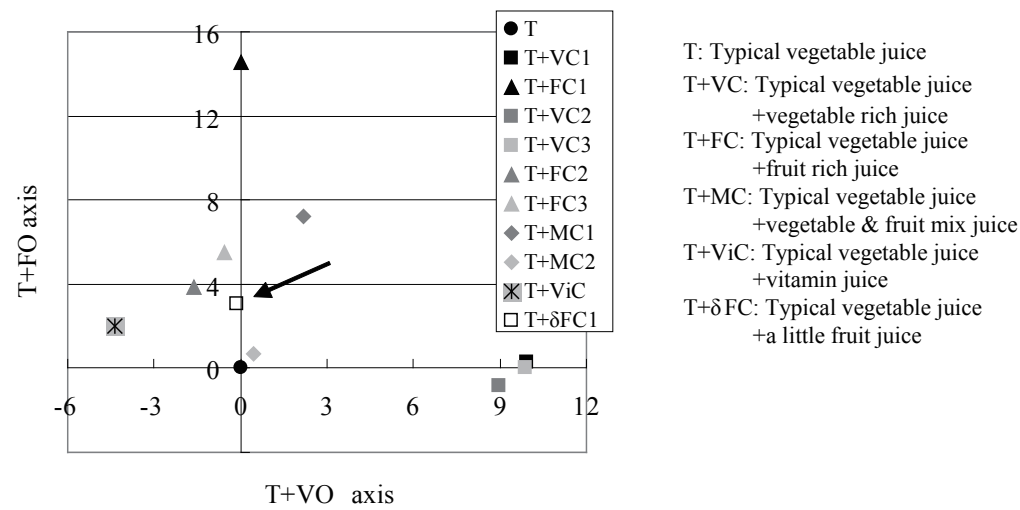

Fig. 11. Odor deviation map of vegetable juice (center, typical vegetable juice; deviation 1, typical + vegetable odor; deviation 2, typical + fruity odor). VC represents samples in the vegetable group and the number after VC $(1,2$, and 3$)$ represents the sample number in the group. FC represents samples in the fruity group. $\delta$ represents the case of a small amount of an FC sample added to the reference sample. MC represents samples in the mixed-odor group. ViC represents a sample in which vitamin juice is mixed with vegetable juice. 
of odor deviation maps can be applied to naturally mixed odors, not only artificial odors and mixed chemical components.

The result for a sample obtained by adding a small amount of a product with a fruity odor to the reference sample is plotted on the ordinate, as indicated by an arrow $(\mathrm{T}+\delta \mathrm{FC})$. This indicates that a substance mixed into the reference sample can be detected. If the number of candidates for the substance mixed into a reference sample is limited, the substance can be estimated by odor deviation mapping, even if it has a multicomponent odor.

\section{Conclusions}

In this study, we developed a method of resolving the problem of the nonlinearity of sensor outputs, which has hampered the practical application of fragrance and flavor analyzers equipped with sensors. As pretreatment systems, a dilution and mixing apparatus and a concentrating system were installed before the sensing stage. The concentrations of samples were adjusted by applying feedback on the basis of sensor outputs with respect to the sample concentrations. Thus, we established a measurement method that can adjust the lengths of vectors comprising multiple sensor outputs for different samples. The repeatability of the measurement, particularly for the case of differences in the type of odor, was improved by this method.

Considering the possibility of calibrating sensors, we developed a technique of odor deviation mapping to express differences in the type of odor by making full use of the measurement method for adjusting the vector length, and we demonstrated its improved repeatability. Figure 12 shows the system that incorporates the method and technique developed in this study. We also discussed the expected applications of this system, such as the quantification and qualification of differences in odor among samples, the determination of expiration dates of foods, the identification of an unusual odor in multicomponent odors, and the preparation of odor deviation maps for commercial products.

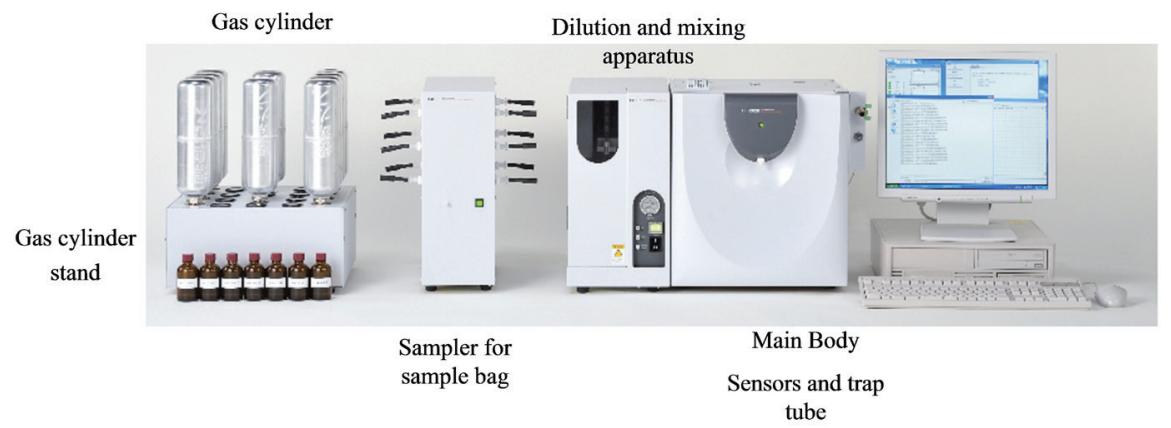

Fig. 12. (Color online) FF-2020 fragrance and flavor analyzer system. 


\section{References}

1 K. C. Persaud and G. H. Dodd: Nature 299 (1982) 352.

2 T. C. Pearce, S. S. Schiffman, H. T. Nagle and J. W. Gardner: Handbook of Machine Olfaction (Willy-VCH, Weinheim, 2003) Chap. 7.

3 S. Sankaran, L. R. Khot and S. Panigrahi: Sens. Actuators, B 171-172 (2012) 1.

4 U. Weimer and M. Frank: Proc. 6th Int. Symp. Olfaction \& Electronic Nose 99 and 1st Workshop of the Network of Excellence on Artificial Olfactory Sensing Nose (Institute of Physical Chemistry of the University of Tübingen, Tübingen, 1999).

5 H. V. Shurmer, J. W. Gardner and H. T. Chan: Sens. Actuators 18 (1989) 361.

6 H. Hong, H. Woo Shin, H. Park, D. Yun, C. Kwon, K. Lee, S. Kim and T. Moriizumi: Sens. Actuators, B 33 (1996) 68.

7 C. D. Natale, R. Paolesse, A. Macagnano, A. Mantani, A. D'Amico, A. Legin, L. Lvova, A. Rudnitskaya and Y. Vlasov: Sens. Actuators, B 64 (2000) 15.

8 P. M. Schweizer-Berberich, S. Vaihinger and W. Göpel: Sens. Actuators, B 18-19 (1994) 282.

9 J. M. Slater, J. Payntter and J. Watt: Analyst 118 (1993) 379.

10 H. V. Shumer and J. W. Gardner: Sens. Actuators, B 8 (1992) 1.

11 J. W. Gardner, H. V. Shumer and T. T. Tan: Sens. Actuators, B 6 (1992) 71.

12 M. A. Craven, J. W. Gardner and P. N. Bartlett: Trends Anal. Chem. 15 (1996) 486.

13 J. Kita, Y. Aoyama, M. Kinoshita, H. Akamaru and M. Okada: Shimadzu Review 64 (2007) 63 (in Japanese).

14 M. Meilgaard: MBBA Tech. Q. 28 (1991) 132.

\section{About the Authors}

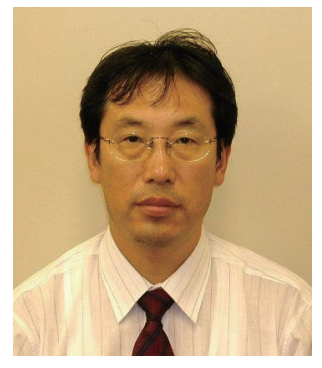

Junichi Kita received his bachelor's degree in 1984 from the Department of Chemical Engineering, Kyoto University. After that, he joined Shimadzu Corporation in Kyoto and studied sensor fields such as infrared sensors, high-temperature superconductive magnetic sensors, and odor sensors consisting of conducting polymers and metal oxide semiconductors. Finally, he developed electronic nose devices and he will receive his $\mathrm{Ph} . \mathrm{D}$. degree from Kyushu University in March, 2014. He is a member of the Institute of Electrical Engineers of Japan, the Electrochemical Society of Japan, and the Japan Association on Odor Environment.

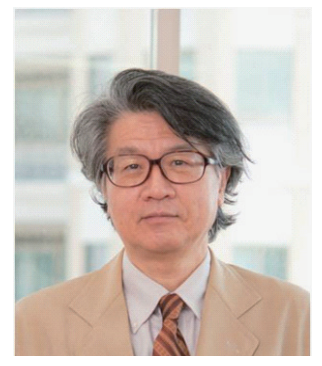

Kiyoshi Toko was born in Fukuoka, Japan, in 1953. He received his B.E., M.E., and Ph.D. degrees in electrical engineering from Kyushu University, Fukuoka, in 1975, 1977, and 1982, respectively. He was the Dean of the Graduate School of Information Science and Electrical Engineering, Kyushu University, from 2008 to 2011, and he is currently a Distinguished Professor. He is a member of the Japan Society of Applied Physics, the Institute of Electrical Engineers of Japan, and the Japanese Association for the Study of Taste and Smell. 